\title{
Probing the Oncolytic and Chemosensitizing Effects of Dihydrotanshinone in an In Vitro Glioblastoma Model
}

\author{
VARUN KUMAR $^{1}$, DANIEL RADIN ${ }^{2}$ and DONNA LEONARDI ${ }^{1}$ \\ ${ }^{1}$ Bergen County Academies, Hackensack, NJ, U.S.A.; \\ ${ }^{2}$ Department of Pharmacology, Stony Brook University School of Medicine, Stony Brook, NY, U.S.A.
}

\begin{abstract}
Background: Temozolomide is the primary chemotherapeutic agent used to treat glioblastoma. However, many tumors are initially resistant to or develop resistance to temozolomide, mainly due to high levels of $O^{6}$-methylguanine DNA transferase (MGMT) which repairs DNA damage traditionally caused by temozolomide. Dihydrotanshinone (DHT) is extracted from Salvia miltiorrhiza, a Chinese medicinal plant, and has also been shown to have antiproliferative effects on various cancer cell lines. DHT has been to shown to induce apoptosis via induction endoplasmic reticulum stress, that can reportedly sensitize cells to temozolomide. Materials and Methods: MTS cellular proliferation assays or trypan blue viability assays were used to determine the effects of DHT/temozolomide combinatorial treatment. Enzyme-linked immunosorbent assay (ELISA) was used to determine effects on MGMT and P-glycoprotein levels after singular and combinatorial treatment. Results: DHT had a synergistic oncolytic effect in a MGMT-deficient cell line and a sensitizing effect in a MGMT-expressing cell line. Cytotoxicity due to DHT was shown to be reactive oxygen species-dependent, while the combinatorial effect of DHT and temozolomide synergistically reduced MGMT and P-glycoprotein levels. Conclusion: DHT was shown to augment temozolomide efficacy, indicating that, since DHT can penetrate the blood-brain barrier, temozolomide in combination with DHT may represent a promising therapeutic option for glioblastoma.
\end{abstract}

This article is freely accessible online.

Correspondence to: Daniel Radin, Stony Brook University School of Medicine, Stony Brook, 11790, NY, U.S.A. Tel: +1 2016790671, Fax: +1 2014930887, e-mail: danradin1@gmail.com and Varun Kumar, Bergen County Academies, Hackensack, NJ, U.S.A. E-mail: varkum@outlook.com

Key Words: Dihydrotanshinone, drug resistance, glioblastoma, MGMT, synergy, temozolomide.
Glioblastoma (GBM) is the most common and aggressive type of brain tumor in adults. Despite multimodal treatment, the median survival rate is $14-16$ months, with about a $2 \% 3$-year survival rate $(1,2)$. GBM is characterized by extremely invasive capabilities, highly destructive malignancy, and a highly increased proliferative rate. GBM is commonly treated with temozolomide, but many tumors are initially resistant to or develop resistance to temozolomide treatment (3). It is therefore necessary to find a treatment complementary to temozolomide.

Temozolomide induces cytotoxicity in GBM cells by DNA methylation of guanine at the $O^{6}$ position. The $O^{6}$ methylguanine binds incorrectly with thymine, which triggers the mismatch repair system, leading to cell-cycle arrest and apoptosis (4). Commonly, in temozolomide resistance, $O^{6}$ methylguanine DNA transferase (MGMT) repairs the methylation of guanine at the $O^{6}$ position, thus rendering temozolomide incapable of inducing apoptosis (5). Additionally, up-regulation of the drug efflux pump Pglycoprotein has been linked to temozolomide resistance (6).

Dihydrotanshinone (DHT) is a component of the traditional Chinese medicinal plant Salvia miltiorrhiza. DHT is a therapeutic agent for Alzheimer's, cardiovascular disease, hepatitis, inflammation, and cancer (7-10). For instance, DHT inhibited the expression of nuclear factor kappa B and potentiated tumor necrosis factor alpha-induced apoptosis in cervical cancer (11). DHT was also found to reverse multidrug resistance by modulating P-glycoprotein expression in colon cancer (12). Moreover, out of the abietane diterpenes extracted from $S$. miltiorrhiza, DHT most potently penetrates the blood-brain barrier (BBB) (7).

Given that DHT has oncolytic potential and is known to pass the BBB, we sought to investigate potential oncolytic effects of this drug in established GBM cell lines that express MGMT (T98G) and do not express MGMT (U87) (3). Additionally, since DHT induces endoplasmic reticulum (ER) stress (13) and drugs that induce ER stress have been shown to sensitize cells to temozolomide (14), we also investigated the effects of treating GBM cells with this drug in the presence of temozolomide, a front-line therapy for GBM. 


\section{Materials and Methods}

Cell culture. U87-MG and T98G human glioblastoma cell lines were acquired from the American Type Culture Collection (\#HTB14 \& CRL-1690; ATCC, Manassas, VA, USA). Cells were grown in Dulbecco's modified Eagle medium (DMEM) with Glutamax (ThermoFisher, Waltham, MA, USA) supplemented with $10 \%$ fetal bovine serum (FBS) (Gibco, Waltham, MA, USA) and 1\% 5,000 $\mathrm{U} / \mathrm{ml}$ Penn/Strep (ThermoFisher). C8-D1A, a mouse astrocyte cell line (ATCC, \#CRL-2541), was grown in DMEM with FBS, Glutamax, and Penn/Strep additionally supplemented with $1 \% \mathrm{~N}-2$ supplement (ThermoFisher). Cells were incubated at $37^{\circ} \mathrm{C}$ with $5 \%$ $\mathrm{CO}_{2}$ and $100 \%$ humidity.

Reagents. DHT (Sigma-Aldrich, St. Louis, MO, USA) was dissolved in dimethylsulfoxide. Temozolomide (TCI America, Portland, OR, USA) and L-glutathione (Sigma-Aldrich) were freshly dissolved in culture medium.

MTS assay. To determine the effects of combinatorial treatment compared to treatment with either drug alone, proliferation was measured using Cell Titer $96^{\circledR}$ aqueous one solution cell proliferation assay (Promega, Madison, WI, USA). Cells were seeded at 2,500 cells/well and incubated for $24 \mathrm{~h}$ prior to treatment in a 96-well plate. Cells were subsequently treated with 100-400 $\mu \mathrm{M}$ temozolomide, 1-2.5 $\mu \mathrm{M}$ DHT, or 100-400 $\mu \mathrm{M}$ temozolomide plus $1.25 \mu \mathrm{M}$ DHT. After treatment for $72 \mathrm{~h}, 15 \mu \mathrm{L}$ of the MTS reagent was added to each well and the plate was incubated for $1.5 \mathrm{~h}$ before acquiring data at $490 \mathrm{~nm}$ on an ELx808 microplate reader (BioTek, Winnoski, VT, USA). In experiments using glutathione, cells were seeded at 10,000 cells/well and incubated overnight. Cells were then pretreated with $1 \mathrm{mM}$ glutathione for $1 \mathrm{~h}$ and subsequently treated with $2.5 \mu \mathrm{M}$ DHT. After incubation for $24 \mathrm{~h}$, cell proliferation was measured as described above.

Trypan blue exclusion assay. In order to determine the effects of combinatorial treatment compared to treatment with either drug alone, viability was measured using Trypan Blue Exclusion Assay. Cells were seeded at 62,500 cells/well $24 \mathrm{~h}$ prior to treatment in a 6 -well plate. Cells were subsequently treated for $72 \mathrm{~h}$, supernatants were collected, and cells were trypsinized and counted using ViCell XR (Beckman Coulter, Indianapolis, IN, USA).

Preparation of lysates. Protocol was adapted from (15). Briefly, phosphate-buffered saline (Gibco), lysis buffer (Cell Signaling Technology, Danvers, MA, USA), and protease inhibitor cocktail (Sigma-Aldrich) were placed on ice. For each treatment group, $1 \times 10^{6}$ cells were transferred to a $15 \mathrm{ml}$ conical tube. The cells were then spun at 1,200 rpm for $7 \mathrm{~min}$ to pellet. Cells were washed with $11 \mathrm{ml}$ of ice cold $1 \times$ PBS. Cells were moved to a microcentrifuge tube and re-centrifuged at $1,000 \mathrm{rpm}$ for $7 \mathrm{~min}$. PBS was decanted. $1 \mathrm{ml}$ of $1 \times 1$ lysis buffer was added $(10 \mu \mathrm{l}$ of protease inhibitor cocktail was added immediately prior to lysis) for $10 \mathrm{~min}$ on ice. Cells were centrifuged at $13,000 \mathrm{rpm}$ for $15 \mathrm{~min}$ and the clear supernatant was moved to a pre-chilled microcentrifuge tube. Samples were stored at $80^{\circ} \mathrm{C}$.

Indirect enzyme-linked immunosorbent assay. Lysates were plated in a 96-well plate for $24 \mathrm{~h}$ at $4^{\circ} \mathrm{C}$ and then removed by aspiration and $300 \mu \mathrm{L}$ Subsequently, $100 \mu \mathrm{L}$ of MGMT Rabbit anti-Human
Primary Antibody (Origene, Rockville, MD, USA) in BSA buffer was then added to each well for $1 \mathrm{~h}$ at $25^{\circ} \mathrm{C}$ then $100 \mu$ Lof Goat Anti-Rabbit Secondary Antibody (Seracare) in BSA buffer was added to each well for $1 \mathrm{~h}$ at $25^{\circ} \mathrm{C}$. One hundred microlitres of ATBS Substrate Solution (Seracare) was added to each well and the plate was incubated at $25^{\circ} \mathrm{C}$ until it developed. The absorbance was read at $405 \mathrm{~nm}$ (ELx808 Biotek). The plate was washed with wash buffer at least three times between steps.

Data analysis. Data were analyzed using Microsoft Excel 2016 (Microsoft, Redmond, WA, USA) and Statplus software (Microsoft, USA) to perform ANOVA. A Student's $t$-test was used to determine statistical significance (alpha set at 0.05). To determine synergy, King's synergy test was used $(\mathrm{KS}>1.15)$ (16). Kings synergy values were determined by dividing the fractional effect of both drugs $(\mathrm{AB})$ by the individual effect of each drug while accounting for assumed additive effects $[\mathrm{A}+\mathrm{B}-(\mathrm{A} \times \mathrm{B})]$.

\section{Results}

DHT displays oncolytic effects in MGMT-expressing and -deficient cell lines. We initially began our investigation by treating two glioblastoma cell lines, T98G (MGMT-positive) and U87 (MGMT-negative), along with healthy mouse astrocytes with increasing doses of DHT. We determined that DHT dose-dependently reduced the viability of both GBM lines (ANOVA, $p<0.001$ ) after 72-h exposure (Figure 1A). DHT was also toxic to non-transformed astrocytes, although they were slightly yet significantly more resistant to DHT, as evidenced by a higher half-maximal inhibitory concentration $\left(\mathrm{IC}_{50}\right)$ value $(2.33 \mu \mathrm{M})$. Subsequently, we asked whether ROS are involved in the oncolytic effect of DHT. To that end, we pre-treated T98G cells with Lglutathione, an antioxidant, prior to administering DHT for $24 \mathrm{~h}$. Glutathione pre-treatment significantly prevented DHTinduced cytotoxicity in T98G cells (Figure 1B, $p<0.001$ ), implicating the generation of ROS as a significant mediator of DHT-mediated toxicity in glioblastoma cells.

DHT reverses temozolomide resistance in an MGMTexpressing cell line. T98G cells were simultaneously treated with a combination of $1.25 \mu \mathrm{M}$ DHT and 100-400 $\mu \mathrm{M}$ temozolomide for $72 \mathrm{~h}$ revealing a reduction of cell viability greater than the effects of the two drugs alone (Figure 2A), suggesting that combinatorial treatment can bypass DNAdamage repair by MGMT. Furthermore, treatment with DHT and temozolomide produced a synergistic effect at each concentration of temozolomide used (Figure 2A) as clarified with a trypan blue exclusion viability assay, which revealed a synergistic increase in the number of dead cells (Figure 2B).

DHT and high-dose temozolomide exert a synergistic antiproliferative effect in an MGMT-deficient cell line. We continued our investigation with U87 cells. It was found that combinatorial treatment with DHT and temozolomide 
A

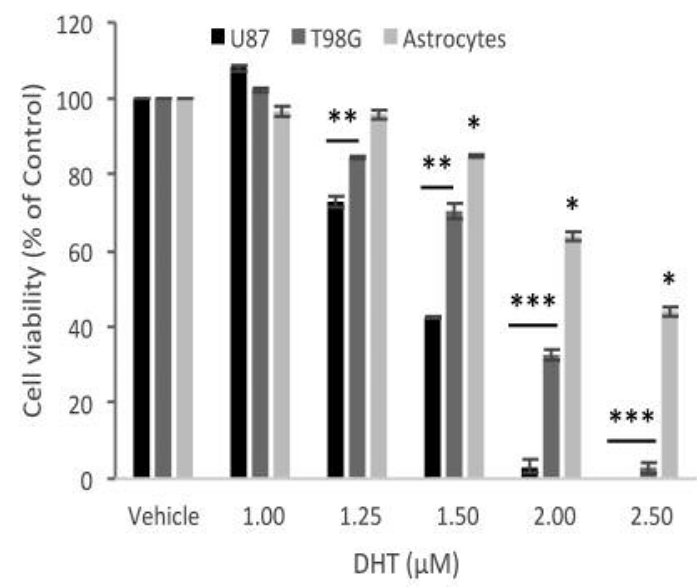

B

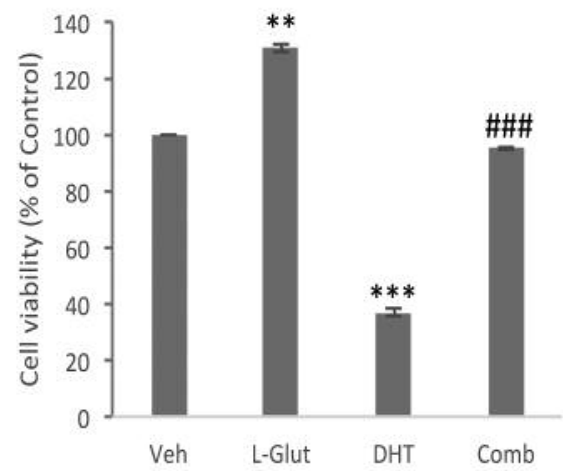

Figure 1. A: Effects of dihydrotanshinone (DHT) on glioblastoma cells and astrocytes. DHT was found to be slightly more toxic toward glioblastoma cells than healthy astrocytes, independent of $O^{6}$-methylguanine DNA transferase (MGMT) status. B: DHT cytotoxicity is reactive oxygen species-dependent. Pretreatment with the antioxidant glutathione prevented DHT-induced cell death. Results from two to three separate experiments are shown as the mean $\pm S E M(n=5)$. Significantly different by $t$-test at $* p<0.05, * * p<0.01$ and $* * * p<0.001$ comparing data to that of the vehicle

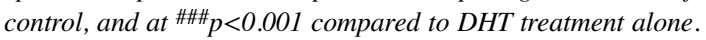

inhibited proliferation more effectively than both of the drugs alone at higher concentrations (Figure 3A), indicating that combinatorial treatment is efficacious in both MGMTexpressing and -deficient cell lines. Combinatorial treatment was also found to subtly yet significantly increase the number of dead cells (Figure 3B, $p<0.05$ ).

Combinatorial treatment with DHT and temozolomide synergistically reduced MGMT and P-glycoprotein levels. To investigate the mechanism of action of DHT and

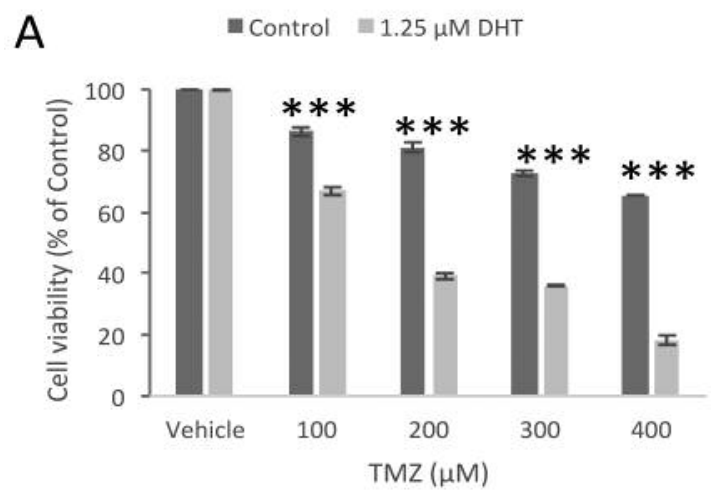

B

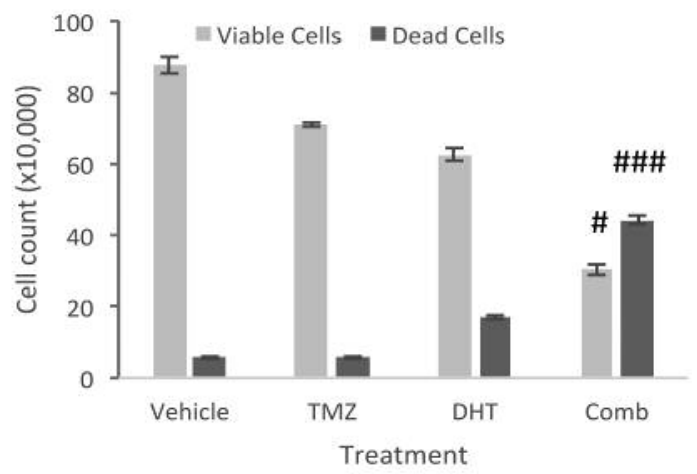

Figure 2. Treatment with dihydrotanshinone (DHT) sensitizes T98G cells to temozolomide (TMZ). A: Concomitant treatment with $1.25 \mu \mathrm{M} \mathrm{DHT}$ and temozolomide markedly reduced the half-maximal inhibitory concentration (IC50) of temozolomide. ***Significantly different by $t$ test at $p<0.001$ comparing data to that at the same temozolomide dose alone. B: Counts of viable and dead cells after treatment with $1.25 \mu \mathrm{M}$ DHT and $200 \mu M$ temozolomide, alone and in combination using the same concentrations. Significant at ${ }^{\#} p<0.05$ and ${ }^{\# \# \#} p<0.001$ by King's synergy test, indicating a significant synergistic interaction between DHT and temozolomide.

combinatorial treatment with DHT and temozolomide, T98G and U87-MG cells were treated with DHT, temozolomide or their combination. After treatment for $72 \mathrm{~h}$, cells were lysed and assayed for changes in MGMT and P-glycoprotein levels (Figure 4). It was found that combinatorial treatment with DHT and temozolomide practically reduced MGMT levels to $50 \%$ that of the vehicle control $(p<0.01$, King's synergy test), suggesting that the interaction of the two drugs pharmacologically modulates the level of MGMT. In addition, it was confirmed that U87-MG is MGMT-deficient (data not shown), indicating that the synergistic inhibition of proliferation in the MGMT-deficient cell line appears to be through a different mechanism compared to the MGMTexpressing cell line. Furthermore, the combination treatment also led to a synergistic reduction of P-glycoprotein levels 


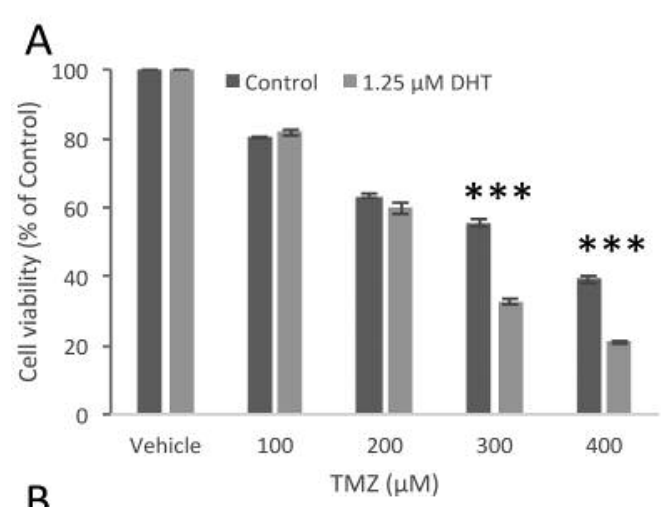

B

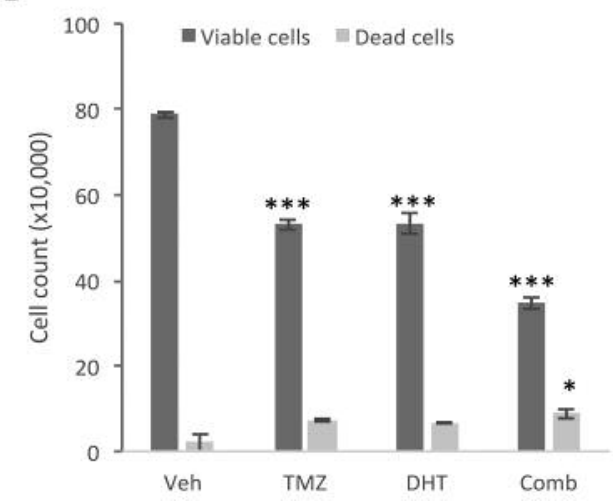

Figure 3. Treatment with dihydrotanshinone (DHT) sensitizes U87 cells to temozolomide (TMZ) at high concentrations. A: Concomitant treatment with DHT and temozolomide markedly reduced the halfmaximal inhibitory concentration $\left(I_{50}\right)$ for temozolomide (refer to Table I). Results from three separate experiments are shown as mean \pm SEM $(n=5)$. Significantly different by t-test at $* * * p<0.001$ comparing data to that of temozolomide alone. B: Counts of viable and dead cells after treatment with $1.25 \mu M$ DHT and $200 \mu M$ temozolomide, alone and in combination at the same concentrations. Significantly different by $t$-test at $* p<0.05, * * * p<0.001$ comparing data to that of the vehicle-treated control.

(Figure 4), demonstrating that the combination strategy, in addition to reducing the level of MGMT, may have increased the intracellular concentration of temozolomide by inhibiting its efflux through P-glycoprotein.

\section{Discussion}

The purpose of this study was to investigate the oncolytic potential of DHT in an in vitro model of glioblastoma and to determine if there were any interactions with temozolomide, a standard-of-care chemotherapeutic agent used to manage GBM clinically. It was previously shown that DHT reverses multidrug resistance by modulation of P-glycoprotein expression and DHT alone has oncolytic effects in various cancer cell lines $(12,17)$. To our

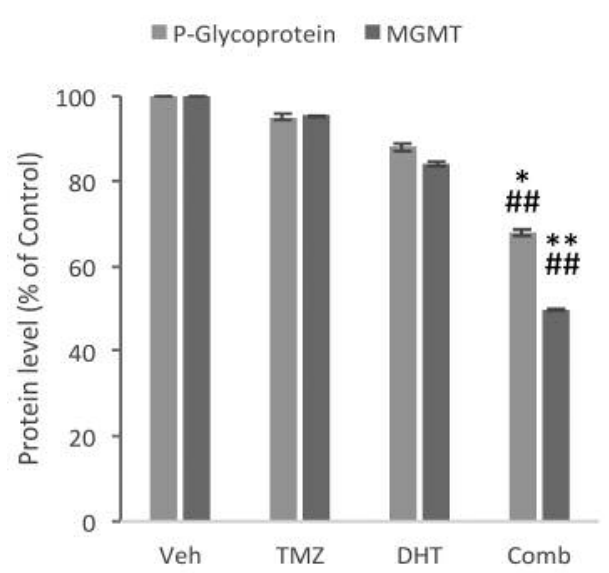

Figure 4. Dihydrotanshinone (DHT) and temozolomide synergistically reduces protein expression of $O^{6}$-methylguanine DNA transferase (MGMT) and P-glycoprotein in T98G cells. Treatment with DHT and temozolomide alone reduced MGMT levels, but treatment with both in combination reduced MGMT levels substantially more than treatment with either drug alone. Results from two separate experiments are shown as mean $\pm \operatorname{SEM}(n=4)$. Significantly different by $t$-test at $* p<0.05$ and ${ }^{* *} p<0.01$ compared to vehicle control. Significantly at ${ }^{\# \#} p<0.01$ by King's synergy test, indicating a significant synergistic interaction between DHT and temozolomide.

knowledge, for the first time, we have demonstrated that DHT has oncolytic effects in a GBM cell line and sensitizes glioblastoma cells to temozolomide treatment.

DHT was shown to induce apoptosis via the induction of ROS $(18,19)$ and subsequent activation of c-Jun $N$-terminal kinase (17). The apoptosis triggered by DHT seems to be p53-independent (17), as seen with other standard chemotherapies, such as paclitaxel and rapamycin $(20,21)$. It was confirmed that DHT cytotoxicity is ROS-dependent even in GBM (Figure 1B), but the specific pathways that DHT modulates in GBM have yet to be fully elucidated. DHT reportedly induces ER stress to trigger apoptosis (8). Importantly, drugs that induce ER stress can sensitize glioma cells to temozolomide treatment (9).

Drug resistance in cancer can be intrinsic to the tumor or it can be acquired as chemotherapy progresses. Resistance to chemotherapeutic agents can be a result of increased drug efflux by efflux pumps, such as by P-glycoprotein (22), as well as a result of overexpression of molecules that directly counteract the drug. Specifically, drug inactivation, drugtarget alteration, DNA-damage repair, and cell-death perturbation play a role in counteracting the effects of chemotherapeutic drugs (23). However, the exact mechanism of action of drug resistance in some cases is unclear, while in other cases it depends on the drug and the type of tumor. 
Table I. Half-maximal inhibitory concentration $\left(I_{50}\right)$ values for cell lines and drugs used in this study. Dihydrotanshinone (DHT) and temozolomide (TMZ) differentially affected U87 $O^{6}$-methylguanine DNA transferase (MGMT)-negative and T98G (MGMT-positive) cells. DHT significantly sensitized T98G and U87 cells to temozolomide treatment.

\begin{tabular}{lccc}
\hline & \multicolumn{3}{c}{$\mathrm{IC}_{50}(\mu \mathrm{M})$} \\
\cline { 2 - 4 } Cell line & DHT & TMZ & $\begin{array}{c}\text { TMZ combined } \\
\text { with } 1.25 \mu \mathrm{M} \text { DHT }\end{array}$ \\
\hline T98G & $1.78 \pm 0.006$ & $626 \pm 16$ & $182 \pm 6^{\# \# \#}$ \\
U87 & $1.50 \pm 0.009 * * *$ & $323 \pm 6$ & $244 \pm 2^{\# \# \#}$ \\
\hline
\end{tabular}

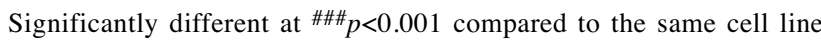
treated with TMZ alone, and at $* * * p<0.001$ comparing values between cell lines.

Temozolomide is most commonly used to treat GBM but many tumors acquire or initially are temozolomide resistant. In both acquired and intrinsic temozolomide resistance, $M G M T$ expression is up-regulated, thus rendering DNA damage by temozolomide ineffective (4). Although not as crucial as MGMT, P-glycoprotein expression contributes to temozolomide resistance because temozolomide is a good substrate of this efflux pump (6). Mechanisms of intrinsic resistance are seemingly different from mechanisms of acquired resistance, although they share various key similarities (3). In acquired resistance, NF-kB and signal transducer and activator of transcription 3 (STAT3) are often modulated in some cell lines, while in intrinsic resistance, p53 can be modulated in some cell lines (3). This makes gene therapy targeting one specific gene in temozolomideresistant GBM less feasible than a pharmacological drug that has various effects in tumors of different genotypes.

Our results collectively demonstrate that treatment with DHT sensitized cells to temozolomide and complemented temozolomide in producing an oncolytic effect. DHT sensitized temozolomide resistant cells to temozolomide in a synergistic fashion (Figure 2A, Table I), while producing a synergistic effect in temozolomide-sensitive cells at higher doses of temozolomide (Figure 3A). It was found that sensitization occurred via downregulation of protein expression of MGMT and P-glycoprotein (Figure 4). It is likely that the phosphoinositide 3-kinase/protein kinase $\mathrm{B}$ (PI3K/AKT) pathway is upstream in the pharmacological modulation of MGMT (24). How the interaction of DHT and temozolomide produces a synergistic decrease in MGMT and P-glycoprotein levels has yet to be investigated. If temozolomide and DHT synergistically down-regulate the P-glycoprotein level in MGMT-deficient cells, this may also explain the synergistic effect of the dual-drug regimen at higher doses of temozolomide. Additionally, ER stress could play a role in complementing temozolomide to inhibit glioblastoma cell proliferation (21), although we speculate the reduction of MGMT is far more important in sensitization to temozolomide.

DHT has the potential to become a pan-therapeutic option for the treatment of GBM, since its efficacy is not limited to certain types of tumor. It acts via different mechanisms of action to produce synergistic effects and is able to penetrate the BBB, making clinical application of the drug foreseeable in the future.

\section{Conflicts of Interest}

The Authors declare that there are no conflicts of interest in regard to this work.

\section{Acknowledgements}

The Authors would like to acknowledge the Bergen County Academies for providing the funds necessary to carry out this study and Mrs. Megan Schmidt for critical review of this article.

\section{References}

1 Lu L, Li C, Li D, Wang W, Zhou C, Shao W, Peng J, You Y, Zhang $\mathrm{X}$ and Shen $\mathrm{X}$ : Cryptotanshinone inhibits human glioma cell proliferation by suppressing STAT3 signaling. Mol Cell Biochem 381: 273-282, 2013.

2 Walid MS: Prognostic factors for long-term survival after glioblastoma. Perm J 12: 45-48, 2008.

3 Lee SY: Temozolomide resistance in glioblastoma multiforme. Genes Dis 3: 198-210, 2016.

4 Kohsaka S, Wang L, Yachi K, Mahabir R, Narita T, Itoh T, Tanino M, Kimura T, Nishihara $\mathrm{H}$ and Tanaka S: STAT3 inhibition overcomes temozolomide resistance in glioblastoma by downregulating MGMT expression. Mol Cancer Ther 11: 1289-1299, 2012.

5 Kitange GJ, Carlson BL, Schroeder MA, Grogan PT, Lamont JD, Decker PA, Wu W, James CD and Sarkaria JN: Induction of MGMT expression is associated with temozolomide resistance in glioblastoma xenografts. Neuro Oncol 11: 281-291, 2009.

6 Munoz JL, Walker ND, Scotto KW and Rameshwar P: Temozolomide competes for P-glycoprotein and contributes to chemoresistance in glioblastoma cells. Cancer Lett 367: 69-75, 2015.

7 Ren Y, Houghton PJ, Hider RC and Howes MJ: Novel diterpenoid acetylcholinesterase inhibitors from Salvia miltiorhiza. Planta Med 70: 201-204, 2004.

8 Park JW, Lee SH, Yang MK, Lee JJ, Song MJ, Ryu SY, Chung HJ, Won HS, Lee CS, Kwon SH, Yun YP, WS Choi and Shin HS: 15,16-dihydrotanshinone I, a major component from Salvia miltiorrhiza Bunge (Dansham), inhibits rabbit platelet aggregation by suppressing intracellular calcium mobilization. Arch Pharm Res 31: 47-53, 2008.

9 Zhou L, Zuo Z and Chow MS: Danshen: an overview of its chemistry, pharmacology, pharmacokinetics, and clinical use. $\mathrm{J}$ Clin Pharmacol 45: 1345-1359, 2005.

10 Cheng R, Chen J, Wang Y, Ge Y, Huang Z and Zhang G: Dihydrotanshinone induces apoptosis of SGC7901 and MGC803 cells via activation of JNK and p38 signalling pathways. Pharm Biol 54: 3019-3025, 2016. 
11 Wang F, Ma J, Wang KS, Mi C, Lee JJ and Jin X: Blockade of TNF- $\alpha$-induced NF-kB signaling pathway and anti-cancer therapeutic response of dihydrotanshinone $\mathrm{I}$. Int Immunopharmacol 28: 764-772, 2015.

$12 \mathrm{Hu}$ T, To KK, Wang L, Zhang L, Lu L, Shen J, Chan RL, Li M, Yeung JH and Cho CH: Reversal of P-glycoprotein (P-gp)mediated multidrug resistance in colon cancer cells by cryptotanshinone and dihydrotanshinone of Salvia miltiorrhiza. Phytomedicine 21: 1264-1272, 2014.

13 Chuang MT, Ho FM, Wu CC, Zhuang SY, Lin SY, Suk FM and Liang YC: 15,16-Dihydrotanshinone I, a compound of Salvia miltiorrhiza Bunge, induces apoptosis through inducing endoplasmic reticular stress in human prostate carcinoma cells. Evid Based Complement Alternat Med 2011: 1-9, 2011.

14 Xipell E, Aragón T, Martínez-Velez N, Vera B, Idoate MA, Martínez-Irujo JJ, Garzón AG, Gonzalez-Huarriz M, Acanda AM, Jones C, Lang FF, Fueyo J, Gomez-Manzano C and Alonso MM: Endoplasmic reticulum stress-inducing drugs sensitize glioma cells to temozolomide through downregulation of MGMT, MPG, and Rad51. Neuro Oncol 18: 1109-1119, 2016.

15 Radin D, Lippa A, Patel P and Leonardi D: Lifeguard inhibition of Fas-mediated apoptosis: A possible mechanism for explaining the cisplatin resistance of triple-negative breast cancer cells. Biomed Pharmacother 77: 161-166, 2016.

16 Han L, Wu J and Yang L: Effect of combination of rapamycin and cisplatin on human cervical carcinoma hela cells. Contemp Oncol 16: 512-515, 2012.

17 Sung B, Chung H, Kim M, Kang Y, Kim D, Hwang S, Kim M, Kim C, Chung $\mathrm{H}$ and Kim N: Cytotoxic effects of solventextracted active components of Salvia miltiorrhiza Bunge on human cancer cell lines. Exp Ther Med 9: 1421-1428, 2015.
18 Wang L, Yeung JH, Hu T, Lee WY, Lu L, Zhang L, Shen J, Chan $\mathrm{RL}, \mathrm{Wu} \mathrm{WK}$ and Cho CH: Dihydrotanshinone induces p53independent but ROS-dependent apoptosis in colon cancer cells. Life Sci 93: 344-351, 2013.

19 Lee WY, Liu KW and Yeung JH: Reactive oxygen speciesmediated kinase activation by dihydrotanshinone in tanshinonesinduced apoptosis in HepG2 cells. Cancer Lett 285: 46-57, 2009.

20 Woods CM, Zhu J, McQueney PA, Bollag D and Lazarides E: Taxol-induced mitotic block triggers rapid onset of a p53independent apoptotic pathway. Mol Med 1: 506-526, 1995.

21 Miyake N, Chikumi H, Takata M, Nakamoto M, Igishi T and Shimizu E: Rapamycin induces p53-independent apoptosis through the mitochondrial pathway in non-small cell lung cancer cells. Oncol Rep 28: 848-854, 2012.

22 Gottesman MM: Mechanisms of cancer drug resistance. Annu Rev Med 53: 615-627, 2002.

23 Housman G, Byler S, Heerboth S, Lapinska K, Longacre M, Snyder N and Sarkar S: Drug resistance in cancer: an overview. Cancers 1769-1792, 2014.

24 Zhang LH, Yin AA, Cheng JX, Huang HY, Li XM, Zhang YQ, Han $N$ and Zhang X: TRIM24 promotes glioma progression and enhances chemoresistance through activation of the PI3K/AKT signaling pathway. Oncogene 34: 600-610, 2015.
Received August 25, 2017

Revised September 25, 2017 Accepted September 27, 2017 